\title{
PW03-025 - Procaspase-1 contributes to inflammation via NF-KB
}

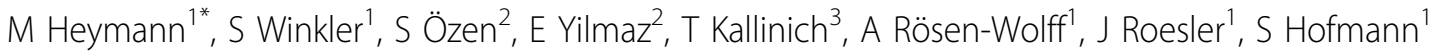 \\ From 7th Congress of International Society of Systemic Auto-Inflammatory Diseases (ISSAID) \\ Lausanne, Switerland. 22-26 May 2013
}

\section{Introduction}

Caspase-1 is a pro-inflammatory enzyme which gets activated by autoprocessing following the assembly of multiprotein complexes called inflammasomes. Mature caspase-1 is responsible for the activation of the proinflammatory cytokines interleukin (IL)- $1 \beta$ and IL-18. Luksch and colleagues reported naturally occurring CASP1 genetic variants in patients suffering from unexplained recurrent febrile episodes. Paradoxically, in vitro and in vivo analyses revealed decreased enzymatic activity of these caspase-1 variants leading to impaired cytokine production. A study of Lamkanfi and colleagues provides a possible explanation by indicating a link between enzymatically inactive procaspase- 1 and activation of NF- $\kappa \mathrm{B}$, a pro-inflammatory transcription factor.

\section{Objectives}

We tried to solve the indicated paradox by analyzing NF- $\kappa \mathrm{B}$ activation in the presence of the procaspase- 1 variants found.

\section{Methods}

NF- $\kappa \mathrm{B}$ activity was determined using a luciferase reporter assay system in transfected HEK 293T cells. RIP2 cleavage and ubiquitination studies were also performed in these cells. Protein/protein interactions of RIP2 and procaspase- 1 were investigated in THP-1 cells by coimmunoprecipitation and in human monocyte derived macrophages by confocal fluorescence microscopy.

\section{Results}

Procaspase-1 variants with reduced enzymatic activity increased NF- $\kappa \mathrm{B}$ activation by interacting with RIP2 (receptor interacting protein kinase 2). In contrast, wildtype (wt) procaspase-1 reduced NF- $\kappa \mathrm{B}$ activity by cleaving RIP2 and decreasing RIP2 ubiquitination which is essential for NF- $\kappa \mathrm{B}$ activation. In addition to transfection experiments, we showed RIP2/procaspase-1 interaction in the human monocyte cell line THP-1 and in human monocyte derived macrophages after stimulation with LPS in a time dependent manner.

\section{Conclusion}

Our results support the hypothesis that procaspase-1 variants with reduced enzymatic activity bind to RIP2 and thereby increase NF- $\kappa \mathrm{B}$ activitation. This may contribute to pro-inflammatory signalling and thereby contribute to unexplained recurrent febrile episodes in the patients.

\section{Disclosure of interest}

None declared.

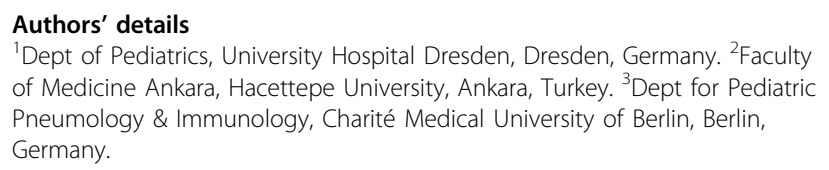
of Medicine Ankara, Hacettepe University, Ankara, Turkey. ${ }^{3}$ Dept for Pediatric Pneumology \& Immunology, Charité Medical University of Berlin, Berlin, Germany.

Published: 8 November 2013

doi:10.1186/1546-0096-11-S1-A251

Cite this article as: Heymann et al:: PW03-025 - Procaspase-1

contributes to inflammation via NF-KB. Pediatric Rheumatology 201311 (Suppl 1):A251. 